\title{
L'idea di finzione nella letteratura francese degli anni Venti: Montherlant e Cocteau
}

\section{Pierangela Adinolfi}

\section{Q OpenEdition}

1 Journals

\section{Edizione digitale}

URL: http://journals.openedition.org/studifrancesi/9103

DOI: ERREUR PDO dans /localdata/www-bin/Core/Core/Db/Db.class.php L.34 : SQLSTATE[HYO00]

[2006] MySQL server has gone away

ISSN: 2421-5856

\section{Editore}

Rosenberg \& Sellier

\section{Edizione cartacea}

Data di pubblicazione: 1 juin 2008

Paginazione: 147-156

ISSN: 0039-2944

\section{Notizia bibliografica digitale}

Pierangela Adinolfi, «L'idea di finzione nella letteratura francese degli anni Venti: Montherlant e Cocteau», Studi Francesi [Online], 154 (LII | I) | 2008, online dal 30 novembre 2015, consultato il 07 janvier 2021. URL: http://journals.openedition.org/studifrancesi/9103; DOI: https://doi.org/ERREUR PDO dans /localdata/www-bin/Core/Core/Db/Db.class.php L.34 : SQLSTATE[HY000] [2006] MySQL server has gone away

\section{(c) (i) (9)}

Studi Francesi è distribuita con Licenza Creative Commons Attribuzione - Non commerciale - Non opere derivate 4.0 Internazionale. 


\title{
Lidea di finzione nella letteratura francese degli anni Venti: Montherlant e Cocteau
}

\author{
«Je suis un mensonge qui dit toujours la vérité». \\ Jean Cocteau, Journal d'un Inconnu.
}

I fenomeni culturali che caratterizzano gli anni Venti sono già rintracciabili, in larga misura, nel periodo pre-bellico. Si tratta dei nuovi orientamenti antipositivistici della filosofia, del richiamo della dimensione inconscia e irrazionale dell'essere umano, del culto dell' "io" e della crisi dell'individualità che conduce irreparabilmente alla disgregazione della personalità. Tra il 1913 ed il 1914, gli scritti di Rivière, Proust, Alain-Fournier, Gide tendono a dissipare le certezze naturaliste e gli artifici simbolisti. Il romanzo francese dell'«entre-deux-guerres» è, tuttavia, fortemente segnato dagli anni della guerra che esasperano le manifestazioni soggettive e le conseguenze oggettive del disagio umano. Numerosi scrittori, in epoche differenti, sono stati ispirati dal clima del conflitto, da Radiguet, Montherlant, Maurois, Cocteau, Kessel, alle rievocazioni più tarde di Drieu La Rochelle, Cendrars, Brasillach, Romains, Vercel, Lacretelle, Aragon. Le temibili ripercussioni del periodo della guerra si riflettono, pertanto, nella letteratura degli anni successivi.

Fra i principali malesseri riscontrabili in questo contesto, va collocato il disagio dell'individuo minacciato nella sua stessa identità dalla precarietà sociale ed emarginato dalla nuova civiltà di massa che lo nega come persona. La solitudine dell' "io" diviene, allora, al contempo fonte di angoscia e di libertà: questo è il punto di partenza dell'individuo che proclama il valore autonomo della sua indipendenza, cercando di affermare se stesso in opposizione alla società che lo esclude. Questo è l'itinerario degli eredi di Barrès: nelle loro prime opere Montherlant e Malraux sfruttano il turbamento individuale della coscienza per ostentare un'inesauribile volontà di potenza. Il romanzo propone così, attraverso personaggi esemplari, percorsi di vita dediti a ricercare volontaristicamente, come alternativa all'inquietudine dell' "io", l'esaltazione del soggetto. Si profila in tal modo la nascita del romanzo del destino o della condizione umana.

Il diffuso sentimento di incertezza e di provvisorietà, dovuto alla frantumazione di un intero sistema di parametri convenzionali, si manifesta attraverso alcuni temi-chiave dell'epoca. Fra questi l'espressione del divario esistente tra verità e mistificazione, essere ed apparire, illusione e realtà: ne sono un alto esempio titoli come Thomas l'imposteur (1923) di Cocteau e Les Faux-monnayeurs (1925) di Gide. Falsari ed impostori, ciascuno dei quali portatore di significato e di caratteristiche proprie ed originali, assumono dignità letteraria. I falsari del romanzo di Gide rappresentano, per esempio, la metafora della progressiva svalutazione dei valori culturali. La verità nell'opera di Gide come in quella di Proust costituisce un traguardo problematico a causa della relatività di ogni punto di vista individuale e dell'auto-inganno di cui si rendono vittime i personaggi protagonisti dei romanzi dei due autori. La corrosione dei valori comporta, alla lunga, incidenze psicologiche e metafisiche, pensiamo al senso soprannaturale rivestito dall'idea di mensonge nell'opera di Bernanos, ma anche questioni essenzialmente etiche. Il problema della falsa rappresentazione della realtà, personale o indotta, l'idea d'impostura lettera- 
ria, contrapposta alla ricerca ed alla trasmissione della verità, diventano lo specchio in cui si riflettono l'amarezza e l'incertezza del periodo del dopoguerra. Testimonianze laiche e testimonianze cattoliche esprimono l'importanza della riflessione sull'idea di finzione in ambito letterario. L'idea d'illusione rousseauiana benefica e consolatoria ${ }^{1}$, cara ai romantici, perde il suo valore esclusivamente positivo per assumere connotazioni sempre più complesse e sfaccettate che traducono il nuovo disagio della condizione umana.

In questo contesto, abbiamo detto, molti sono i romanzi che trattano della guerra, non tutti però lo fanno allo stesso modo. Giono e Céline, per esempio, sono fra coloro che denunciano in maniera esplicita l'atrocità ed il potere distruttivo della guerra. Diverso appare, invece, l'approccio scelto da Montherlant e Cocteau, i quali non si limitano alla tragica descrizione delle esperienze belliche, ma fanno della guerra stessa uno strumento di espressione del sé. Per Montherlant, la guerra è il songe, l'illusione verso cui Alban de Bricoule va incontro ricercando esclusivamente in essa l'esaltazione dell' “io”. Alban come Guillaume, il protagonista di Cocteau, è un personaggio dai tratti autobiografici: Montherlant e Cocteau vissero in prima persona l'esperienza della prima guerra mondiale, ciò che permise loro di trasferire nell'opera letteraria e nella costituzione dei profili umani aspetti concreti derivati dalla realtà. Sia Alban che Guillaume possiedono una concezione personale della guerra. Entrambi rivestono un eroismo che li distanzia dalla società borghese e li oppone alla sua ipocrisia. Alban è il «toucheur d'âmes» che possiede il desiderio assoluto di primeggiare: «Que puis-je contre cette maladie? La prééminence m'est indispensable; rien d'elle ne m'attire; il y a simplement qu'une vie sans elle ne m'est pas concevable» ${ }^{2}$. Nei suoi lunghi colloqui con Prinet, il protagonista del Songe espone il pensiero più profondo di Montherlant relativo all'inutilità delle azioni umane ed alla conseguente e necessaria consapevolezza che ne deriva. Secondo una visione prettamente nichilistica dell'esistenza, la ricerca dell'eccellenza, frutto di un atto di volontà individuale, è per Alban il solo fine che giustifichi la propria vita. Il giovane eroe di Montherlant decide di partire per il fronte per raggiungere l'amico Prinet. La sua azione è determinata esclusivamente da una scelta personale. Alban vede nella guerra la semplicità dell'azione valorosa che conferisce gloria ed onore e che concede il riposo dalla vita sociale e da quella intellettuale, le quali, al contrario, richiedono sforzo e fatica mentale:

[...] Il est nécessaire que je me repose dans l'action. [...] Simplicité de l'action, surtout de l'action de guerre! Pour établir mon influence intellectuelle, sociale, que de patience, de travail, que d'écueils, que d'habileté, - et je serai discuté, pas soutenu par ceux que j'aime; mes meilleurs amis risqueront de devenir mes adversaires; moi-même, qui peut m'assurer que je suis dans le vrai, me délivrer du doute? Mais ici! Se dresser sur un parapet, aller voir et revenir, presser une gâchette, voilà qui est clair, direct, et qui dans l'instant vous donne une grande gloire. Ici les valeurs sont cotées, classées; sur chacune tout le monde est d'accord. Quelle supériorité! Quel repos!

Dalle parole di Alban si può constatare come Montherlant, già a partire dal Songe, romanzo scritto all'età di ventitré anni ma comprensivo di tutte le tematiche che costelleranno l'opera montherlantiana, ponga la distinzione tra una comunità di

(1) Sul tema dell'illusione, segnaliamo l'importante studio di L. SozzI, Il paese delle chimere. Aspetti e momenti dell'idea di illusione nella cultura occidentale, Palermo, Sellerio, 2007, in particolare il capitolo «Il paese delle chimere», pp. 135-182.
(2) H. DE Montherlant, Le Songe, in Romans et Euvres de fiction non théâtrales, Préface par R. SEcrétain, Paris, Gallimard, [1959] 1975 («Bibliothèque de la Pléiade»), pp. 6, 7.

(3) Ibid., pp. 9-10. 
eletti, i soldati che condividono i valori di un codice cavalleresco, mezzo di purificazione e di nobilitazione della guerra, ed il resto della società in cui il dubbio sul valore individuale permane, affaticando e soffocando chi tenti di dissiparlo. Per Alban, la guerra diventa un'occasione in cui ricercare la sfida, in cui trarre piacere dall'ebbrezza dell'emozione estrema. L'atto di volontà coincide col piacere procurato a se stesso: «Je monte en première ligne, rejoindre une compagnie d'infanterie, dans les Hautes-Vosges, pour le plaisir. J'espère que je vais bien m'amuser. C'est égal, un voyage en Espagne m'aurait amusé davantage» ${ }^{4}$. Qui ciò che conta è l'interiorità dell'essere umano. Il campo di battaglia si trasforma nel luogo della ribellione individuale contro i dogmi e i falsi moralismi sociali. Ė nel tempo della guerra che scaturiscono dal soldato le migliori qualità umane: la solidarietà, la lealtà, la tenerezza, il coraggio, il senso dell'onore, la consapevolezza della dignità dell'uomo, come ci ricorderà Montherlant, per esempio, nel Chant funèbre pour les morts de Verdun (1924). Secondo la prospettiva di Alban, la guerra va combattuta per esclusivo convincimento personale, senza diventare vittima di ambigue ideologie politiche che strumentalizzano le popolazioni. In quest'ottica, la guerra è, inoltre, il momento dell'esaltazione della forza fisica maschile, una forza primordiale che si confonde con la potenza della natura e con le potenzialità del pensiero e dell'arte. In ciò risiede per Montherlant la salute del corpo e la purezza dello spirito: «[...] Il y a de la rudesse dans tous les conquérants, conquérants de la guerre ou conquérants de la pensée et de l'art. [...] Il [Alban] est fort et sain, sain jusqu'à l'ingénuité, sain dans toutes ses recherches et toujours pur, à cause de cette santé et de cette force» ${ }^{5}$. Attraverso quest'adesione alle leggi naturali, Alban avverte l'impeto della guerra come il desiderio di eguagliare in bellezza gli elementi esistenti in natura: «Il sentit bien que dans son brusque besoin de la guerre, il y avait l'anxiété que sa vie n'égalât pas en beauté le printemps, qu'elle ne fût pas digne du printemps, que la beauté du printemps fût inemployée et perdue» ${ }^{6}$. Nella ricerca del pericolo estremo e dell'eccellenza dell'azione umana, Alban de Bricoule è come Guillaume Thomas: per entrambi nel pericolo si trova l'esaltazione del senso della vita. Guillaume ritiene che nell'atto eroico si collochi il libero arbitrio. La disubbidienza, la ribellione, il senso assurdo ed eccezionale dell'esistenza sono, per Cocteau, gli elementi propri dell'agire di un personaggio eroico che senza paura si rende artefice del proprio destino come artefice del proprio destino è l'Alban del Songe. Montherlant e Cocteau, attraverso i loro personaggi, inclusa Clémence, figura femminile speculare a quella di Thomas, manifestano lo stesso forte spirito anticonvenzionale che ricorda l'asocialità del Meursault di Albert Camus: «Quand le sifflement descendait sur moi, et qu'en une seconde je pouvais être jeté dans l'éternité, je n'ai eu ni une pensée religieuse, ni un repentir, ni un élan vers aucun de ceux que j'aime»’ ${ }^{7}$. In guerra le regole convenzionali non esistono più. Tutto assume un aspetto straordinario ed è nel pericolo perseguito, in quanto affermazione della prorpria volontà, l'estrema esaltazione dei sensi e dell'istintualità dell'uomo: «Dans le danger qu'on recherche par un acte de volonté, une merveilleuse exaltation de la vie lui [à Alban] venait, parce que là c'est proprement le jeu» ${ }^{8}$. Alban è al fronte soltanto per sua ferma decisione. Egli ha condotto una vita all'insegna del proprio piacere e dei propri desideri. In questo contesto di relativismo e di soggettivismo assoluti anche l'idea della morte perde il suo significato tradizionale e rientra in una prospettiva nichilistica secondo la quale i concetti di vita e di morte si equivalgono: «[...] Je suis ici sur ma demande, par ma décision et mon obstination, et vous appelez ça la fatalité! C’est ma volonté qui constitue la 
fatalité [...]. Mais enfin, que d'histoires autour de la mort! [...] J'ai aimé tout ce qui est élevé; il n'est aucun plaisir que je n'aie connu [...]. Après cela, ça ne me choque pas du tout si je suis obligé d'arrêter là le jeu. J'ai vécu à ma faim»?. Per Guillaume, come vedremo, la morte diventa parte integrante dell'illusione e del gioco ed anche in questo caso il senso ed il valore tradizionali ad essa attribuiti sono perduti.

Nel grande teatro della guerra, lo strumento teorizzato da Alban per far fronte all'ipocrisia e all'inganno della società degli uomini è la feinte. Al tema dell'illusione della guerra si unisce, nel Songe, il tema della finzione in quanto mezzo di salvaguardia della propria individualità e manifestazione della consapevolezza dell'inutilità delle azioni umane:

J'ignore l'utilité de mon sacrifice, et dans le fond je crois que je me sacrifie à quelque chose qui n'est rien, qui est une de ces nuées que je hais. Croyant mon sacrifice inutile, et peut-être insensé, sans témoin, sans désir, renonçant à la vie et à la chère odeur des êtres, je me précipite dans l'indifférence de l'avenir pour la seule fierté d'avoir été si libre. Dans l'Iliade, Diomède se rue sur Énée, bien qu'il sache qu'Apollon rende Énée invulnérable. Hector prédit la ruine de sa patrie, la captivité de sa femme, avant de retourner se battre comme s'il croyait en la victoire. Quand le cheval prophétique annonce à Achille sa mort prochaine: «Je le sais bien», répond le héros, mais au lieu de se croiser les bras et de l'attendre, il se rejette et tue encore d'autres hommes dans la bataille. Ainsi ai-je vécu, sachant la vanité des choses, mais agissant comme si j'en étais dupe, et jouant à faire l'homme pour n'être pas rejeté comme dieu. Oui, perdons-les l'une dans l'autre, mon indifférence et celle de l'avenir! Après avoir feint d'avoir de l'ambition et je n'en avais pas, feint de craindre la mort et je ne la craignais pas, feint de souffrir et je n'ai jamais souffert, feint d'attendre et je n'attendais rien, je mourrais en feignant de croire que ma mort sert, mais persuadé qu'elle ne sert pas et proclamant que tout est juste ${ }^{10}$.

In questo caso la feinte di Alban, nuovo Ettore e nuovo Achille, diventa un mezzo per proteggere la propria libertà di giudizio dalle false convinzioni patriottiche e sociali. Montherlant, come Camus, fa della consapevolezza individuale in merito all'inutilità delle azioni umane uno strumento che fortifica il protagonista del suo romanzo. Alban vive come se ritenesse importante la propria condotta, finge di credere, finge di interessarsi alle cose del mondo pur essendo totalmente cosciente della vanità del tutto. Nel Songe vi è già l'insegnamento che sarà poi enunciato nel Service inutile (1935), in cui la ricerca dell'elevazione morale compiuta dall'essere umano è la stessa che si ritrova nel romanzo pubblicato tredici anni prima: «L'âme dit: service, et l'intelligence complète: inutile» ${ }^{11}$. Questo titolo ossimorico, Service inutile, come altri di Montherlant, ad esempio Chevalerie du Néant ${ }^{12}$, esprime al meglio la necessaria commistione tra l'innalzamento spirituale e lo scetticismo, tra l'aspirazione ad un modello sublime di comportamento ed il concomitante disincanto riguardo all'utilità dei grandi sentimenti. Qual è, pertanto, la saggezza cui approda Montherlant secondo tali convinzioni? Nel corso dell'esistenza terrena per l'uomo è importante rendersi degno delle grandi azioni, pur rimanendo consapevole della loro inutilità. Il bene tratto dall'azione eroica non andrà comunque perduto poiché sarà un piacere che l'individuo avrà procurato a se stesso e quindi alla propria anima, cioè alla parte morale di sé.

(9) Ibid., p. 89.

(10) Ibid., pp. 110-111.

(11) H. DE Montherlant, Service inutile, in Essais, Préface par P. SiPRIOT, Paris, Gallimard, [1963] 1988 («Bibliothèque de la Pléiade»), p. 571.

(12) «[...] La vie est un songe, mais le bien-faire ne s'y perd pas, quelle que soit son inutilité - inutile pour le corps social, inutile pour sauver notre âme, - parce que, ce bien, c'est à nous que nous l'avons fait. C'est nous que nous avons servi [...]» (Cfr. H. de Montherlant, Chevalerie du Néant, in Essais, cit., p. 598). 
Il Songe e la storia di Alban de Bricoule, personaggio che Montherlant tiene a definire autobiografico ${ }^{13}$, rivelano in maniera esplicita la concezione montherlantiana della guerra connessa con l'idea d'illusione: da un lato la guerra, secondo una prospettiva quasi ontologica, è l'esperienza che comprende tutti gli elementi inclusi nelle altre esperienze umane: il tutto ed il nulla, la vita e la morte, il coraggio e la paura, il bene ed il male, la sfida continua, la tenerezza, la solidarietà. Dall'altro lato, la guerra è la grande illusione, l'inganno perpetrato a danno di tutti gli esseri umani inconsapevoli, a discapito della loro libertà. Per Alban-Henry, la feinte è, invece, la simulazione, l'autodifesa nei confronti di un meccanismo che tende a negare le istanze individuali, l'affermazione dell'esigenza di compiere un esclusivo atto di volontà.

Eroe capace di diventare artefice del proprio destino è anche, come abbiamo detto, Guillaume Thomas. Nella sua esigenza del superamento di sé fino alle estreme conseguenze vi è la curiosità fanciullesca di vedere la morte da vicino: in ciò si trova il senso del divertimento, il piacere per il brivido che il ruolo falsamente acquisito gli fa provare. Guillaume crede soltanto all'immagine, al sogno effimero, proprio come un fanciullo crede nei suoi giochi. Si tratta di un personaggio per il quale esiste soltanto ciò che egli crede esistere, soltanto ciò che è frutto del suo pensiero. Thomas vive spontaneamente ai margini del mondo reale, cioè in una finzione soggettiva. Nel titolo del romanzo, Cocteau rappresenta il Tommaso incredulo del Vangelo, che qui diventa, per assurdo, contemporaneamente impostore e cercatore del vero. In realtà, più che impostore, Thomas è un "sonnambulo" per il quale gli altri inventano l'impostura, traendone un profitto personale. A Thomas non resta che lasciar fare: nato Guillaume Thomas ed originario di Fontenoy, si trasforma in Thomas de Fontenoy e passa così per parente del generale omonimo. Guillaume scivola, pertanto, in un mondo le cui leggi non sono più quelle del mondo reale. Egli è stato predisposto all'irrealtà da un «cœur exalté» che si sente costantemente guidato da una stella, seppur menzognera: «[...] Il existe des hommes qui inspirent une confiance aveugle et jouissent de privilèges auxquels ils ne peuvent prétendre. Guillaume Thomas était de cette race bienheureuse. On le croyait. Il n'avait aucune précaution à prendre, aucun calcul à faire. Une étoile de mensonge le menait droit au but» ${ }^{14}$. Thomas è naturalmente creduto dai suoi compagni e da chi lo circonda. In tal modo si trova dotato di uno «charme surnaturel» ${ }^{15}$ abbastanza potente da trascinare coloro che lo avvicinano in quel mondo che, considerato esclusivamente dal punto di vista del buon senso, è pura finzione.

In questo contesto, Cocteau intende "disintossicare" il romanzo dall'illusione della realtà. La guerra, ad esempio, se la si osserva da un certo punto di vista, perde da sola i suoi connotati reali. Solitamente si è abituati a considerare lo sconvolgimento che essa porta nelle vite e nel mondo normale secondo la prospettiva dell'orrore e del patetico. E anche possibile, tuttavia, con una certa predisposizione dell'animo e della mente, essere subito toccati dall'atmosfera d'irrealtà che, a forza di mutare le apparenze, essa sparge su ogni cosa. La parola "disumanità" è il termine che stigmatizza, di solito, le sofferenze e le violenze contro natura che la guerra infligge agli uomini. Ma esiste già una sorta di disumanità nel modificare l'apparenza di un mondo che si era abituati a vedere in una certa maniera, come se fosse stato "umanizzato" dalla costanza della nostra percezione.

(13) Cfr. H. DE MonTHERLant, Va jouer avec cette poussière. Carnets 1958-1964, Paris, Gallimard, 1966, pp. 191-192.

(14) J. Cocteau, Thomas l'imposteur, in Euvres romanesques complètes, Préface par H. GODARD, Édition établie par S. LinARES, Paris, Gallimard, 2006 («Bibliothèque de la Pléiade»), pp. 415, 392.

(15) Ibid., p. 406. 
Sotto lo sguardo di Thomas, come era già avvenuto sotto quello di Apollinaire, la guerra stessa si trova ad essere «déréalisée». Cocteau compie una trasfigurazione del paesaggio della guerra che diventa in tal modo veicolo d'inganno e d'illusione:

Le chef-d'œuvre du secteur, c'étaient les dunes. On se trouvait ému devant ce paysage féminin, lisse, cambré, hanché, couché, rempli d’hommes. Car ces dunes n'étaient désertes qu'en apparence. En réalité, elles n'étaient que trucs, décors, trompe-l'œil, trappes et artifices. La fausse dune du colonel Quinton y faisait un vrai mensonge de femme. [...] En somme, ces dunes aux malices inépuisablement renouvelées, côté pile, présentaient, côté face, aux télescopes allemands, un immense tour de cartes, un bonneteur silencieux ${ }^{16}$.

Come appare evidente, la terminologia qui impiegata da Cocteau è altamente rivelatrice delle idee di artificio e di simulazione insite nel paesaggio della guerra. Il campo di battaglia perde la tragica connotazione del luogo in cui si esercita esclusivamente la violenza per acquisire i tratti immaginifici conferiti dalla mente del protagonista: le dune e le distese di sabbia evocano la seduzione e la bellezza del corpo femminile. Tutto in questo paesaggio induce all'errore e all'autoinganno, tutto è illusione, falsità, apparenza. Attraverso descrizioni di questo genere, Cocteau rivela la completa adesione del suo personaggio con l'illusione che si è creato. L'impostura della guerra è simboleggiata dalle trincee e dalle dune artificiali che rimodellano e plasmano il paesaggio naturale. Thomas come Alban è immerso nel sogno dell'esperienza bellica, ma mentre l'eroe montherlantiano è consapevole dell'inganno e lo rifiuta opponendo il suo scetticismo, Thomas trova la sua completa ed intima realizzazione nella fusione stessa col sogno. Il mondo della guerra è per sua natura un mondo fittizio, destinato ad ingannare il nemico e proprio per tale motivo, nel contesto bellico, è facile scambiare l'apparenza per la realtà, come accade in effetti a Thomas: le metafore diventano la verità delle cose.

Nel romanzo di Cocteau si coglie l'adeguamento del linguaggio alla finzione del soggetto, la partecipazione della parola al gioco dell'impostura, insieme ad un'evidente linearità narrativa che comporta l'appiattimento della descrizione degli eventi: le immagini insostenibili ed ossessive come l'evocazione dei corpi agonizzanti o la visione del giovane tedesco mutilato, privo delle mani, servono ad esprimere l'assurdità della guerra senza manifestare lo sdegno dell'autore. Nonostante l'angosciante realtà in cui è immerso, Thomas dimostra uno spirito divertito e surreale, un tono beffardo che si rivela in conflitto con la drammaticità degli avvenimenti in cui è coinvolto.

Diverso risulta, invece, l'approccio di Montherlant nell'uso della terminologia impiegata per descrivere le situazioni di guerra.

Egli si sofferma, al contrario, con realismo e dovizia di particolari sul racconto degli episodi di guerra con l'intento di palesare un aspetto tragico della vita che entra in contatto diretto con l'individualità del protagonista: la cruenza di certe immagini serve a suscitare la tenerezza e la solidarietà fra i soldati ed Alban ne è sempre direttamente toccato. Le macabre visioni riferite non scivolano sul personaggio principale, ma sono parte integrante della sua esperienza. Ecco, ad esempio, come Montherlant narra l'episodio in cui Alban si rivolge ad un soldato ferito per avere notizie dell'amico Prinet:

- Est-ce qu'il vient de faire l'attaque avec les gars, là?

Les yeux s'étaient fermés, ne parlaient plus. Alban était au-dessus de lui.

- Hein? Est-ce qu'il vient de faire l'attaque? Réponds-moi donc! 
Il sentait affluer dans ses mains les gestes de secouer, de brutaliser cette chair torturée. Soudain les yeux se rouvrirent, la longue main brunâtre, couverte de minuscules éclaboussures de sang, pareilles à des excréments de mouche sur le cadre d'une glace, esquissa un geste vague. Alban sentit sous le pansement la contraction des muscles, l'effort pour parler, le grognement de ce qui n'était plus une bouche. Un peu de bave rosée teinta l'étoffe, autour de la croûte de vieux sang noir. Puis la tête remua un peu, fit signe que non ${ }^{17}$.

Ciò che, pertanto, accomuna i due autori è la realistica e violenta rappresentazione delle dinamiche della guerra, con risvolti che approdano, tuttavia, ad esiti diversi: in Cocteau al consolidamento dello spirito surreale del personaggio, in Montherlant all'ulteriore esaltazione del protagonista attraverso l'elemento tragico.

Per entrambi gli autori, fondamentale è la formulazione dell'inganno delle dinamiche della battaglia: anche per Montherlant, come abbiamo detto, l'illusione della guerra pervade il romanzo: «Il paraît que sans cesse on se trompe, qu'il y a des éclats dont on ne sait même pas qu'on les a reçus, d'autres qu'on a pris pour une simple contusion ${ }^{18}$. Il linguaggio serve ad esprimere l'illusione della guerra in entrambi gli autori, sebbene l'atteggiamento e la consapevolezza dei personaggi riguardo alla finzione siano differenti.

Ma in questo contesto, il linguaggio non è il solo ad essere chiamato in causa. Un sentimento inatteso, di una nuova profondità, affiora in Thomas quando, per sfuggire al fastidio della sua relazione con Henriette, altro motivo, come vedremo, d'inganno e d'illusione, si fa mandare al fronte. La seconda parte del romanzo è caricata di un peso nuovo. Thomas non è più il beneficiario di un errore situato tra malinteso ed impostura. Quasi dimenticato lo slittamento patronimico che aveva dato vita a quell'errore, il personaggio si rivela nella sua vera dimensione. È qui che il profilo psicologico di Thomas assume originalità e grandezza: non si vive come lui una vita al di fuori della vita senza che ciò, alla fine, non approdi alla morte. La nuova forza di Thomas sta nel fatto che egli ne sia consapevole, almeno intuitivamente, e che vi acconsenta. Thomas va incontro ad una morte quasi ricercata poiché il suo destino non potrebbe essere diverso: la sfida alla vita e la coincidenza tra vero e falso hanno raggiunto, nella sua dimensione esistenziale, un livello tale da non permettergli più di riconoscere il limite entro il quale poter salvaguardare la propria sopravvivenza:

Guillaume volait, bondissait, dévalait comme un lièvre.

N'entendant pas de fusillade, il s'arrêta, se retourna, hors d'haleine.

Alors, il sentit un atroce coup de bâton sur la poitrine. Il tomba. Il devenait sourd, aveugle.

«Une balle, se dit-il. Je suis perdu si je ne fais pas semblant d'être mort».

Mais en lui, la fiction et la réalité ne formaient qu'un.

Guillaume Thomas était mort ${ }^{19}$.

Thomas vuol condurre fino in fondo il suo gioco, vuol far finta di essere morto, ma ormai i due ambiti vitali, la «fiction» e la «réalité» sono un tutt'uno: «Guillaume Thomas était mort».

In Guillaume, la finzione e la realtà formano un nucleo indivisibile ed egli ha raggiunto in tale consapevolezza, come nella morte, il luogo ed il tempo della sua verità. Attraverso la verità espressa da Guillaume, Cocteau intende enunciare la propria verità e cioè l'esigenza che per lui s'impone di sfuggire alla alienante mediocrità della vita. Strumento di tale fuga è la creazione artistica che si sviluppa per mezzo di

(17) H. De Montherlant, Le Songe, in Romans, cit., p. 160.

(19) J. Cocteau, Thomas l'imposteur, in Euvres romanesques complètes, cit., p. 430.

(18) Ibid., p. 119. 
molteplici metamorfosi: la finzione e la stravaganza sono i modi in cui, per assurdo, è possibile manifestare ancora qualcosa di autentico nel mondo, la menzogna è quindi, in questo contesto, percepita come unico rivelatore poetico.

L'avanzare verso una fine così contraria al più naturale istinto di sopravvivenza è, pertanto, ciò che fa di Thomas l'imposteur il capolavoro narrativo di Cocteau, poiché palpabile diventa il senso poetico sprigionato dal protagonista. L'impostura proposta da Cocteau sembra essere un antidoto al nulla esistenziale, un'alternativa adeguata che consente di trasferire l'essenza del personaggio su un diverso piano della coscienza individuale. La finzione costituisce, quindi, per Cocteau, la concezione stessa dell'opera d'arte, da rinnovare in continuazione per mezzo di modalità espressive sempre differenti: opera d'arte e vita assumono, in questo contesto, la stessa identità.

Uno dei temi attraverso i quali Montherlant e Cocteau alimentano l'idea di finzione presente nei loro romanzi è quello dell'amore.

Subito si comprende l'effimera natura del «faux amour» esistente fra Alban e Dominique, all'interno di un rapporto non paritario e che vede svantaggiata la figura femminile, colpita nella sua sensibilità di donna: Dominique è per Alban un apprezzabile camarade quando si mette in luce dimostrando l'eccellenza delle sue prestazioni atletiche oppure quando il suo sentimento non minaccia la libertà d'azione e di coscienza del protagonista:

Il y a un an, quand pour la première fois, il l'avait vue, à «Palestra», remuant les genoux dans le mille mètres autour du stade, elle lui avait donné une joie supérieure par la noblesse de sa foulée et la grandeur de son style. Il l'avait désirée, chaude et éperdue d'effort après le poteau, dans le halètement de la victoire ou de la défaite, avec l'ardeur du risque rapporté sur le visage qui n'avait pas rougi. Unique désir d'un jour! Car, lorsqu'il l'avait connue davantage, elle lui avait révélé un sentiment qui à son point parfait excluait le désir, que le désir eût dénaturé et déprécié. Pourquoi dès lors chercher en elle, qui y perdrait, ce plaisir que tant d'autres pouvaient lui donner en y gagnant? $?^{20}$

Ciò che maggiormente affascina Alban è l'amore sensuale, il piacere fisico che deriva dall'incontro dei corpi e nel quale si può trovare il vero lato sublime dell'amore, snaturato, invece, dalla routine e dalla ripetitività insita nella relazione di coppia. L'amore che nutre Dominique non è, quindi, ricambiato allo stesso livello da Alban che traduce la concezione di Montherlant relativa alla reintegrazione del sentimento nell'individualismo, quella particolare concezione secondo la quale soltanto l'amore provato, e non quello ricevuto, garantisce l'autonomia dell'individuo. Anche il sentimento amoroso è, pertanto, in questo contesto, un'illusione dell'animo umano che talvolta si compiace di esserne ingannato. Ciò che determina le azioni di Alban, per il quale «l'amour absolu» non risiede nel possesso continuo dell'oggetto amato, bensì nella diversità e nell'alternanza, è la fonte del suo desiderio verso la quale egli sempre si dirige e per mezzo della quale in lui rinasce la gioia di vivere. Partito per il fronte per sua ferma volontà, demotivato a restarvi dopo la morte di Prinet, Alban de Bricoule decide di ritornare in prima linea perché, senza rimpianto per l'amico perduto, né per l'amore di Dominique, nel suo animo sente rinascere il desiderio dell'«adversité haute» ed avverte l'appartenenza ad un tutto universale che, seppur indifferente, lo comprende nel suo eterno divenire.

Nel romanzo di Cocteau, l'amore di Guillaume per Henriette sembra inizialmente essere uno squarcio di autenticità nel contesto illusorio della guerra: prova ne è il senso di noia avvertito dal giovane ed imputato ai turbamenti del sentimen- 
to amoroso: «Guillaume s'ennuyait, premiers troubles de l'amour» ${ }^{21}$. Il disagio della noia appare qui come un elemento realistico in cui si coglie la verità riservata all'essere umano nel perdurare del rapporto amoroso. L'esigenza di tenere l'interezza della realtà sotto il proprio controllo fa sì che Guillaume metta in atto una nuova impostura, questa volta nei confronti di se stesso: «Au lieu de se dire qu'il aimait Henriette, ce qui sortait de son jeu, il s'hypnotisait sur ce jeu et attribuait son malaise à l'inaction, au manque d'aventures ${ }^{22}$. Thomas è in grado di far vivere l'amore per la principessina soltanto quando la lontananza gli consente d'idealizzare il sentimento attraverso l'immaginazione e la rêverie. Non appena egli rivede la fanciulla, l'intensità dell'amore si spegne a contatto con la realtà. Perché l'amore perduri, Henriette deve rimanere un'apparizione, un oggetto irreale, carico di fascino e di seduzione: «Un miracle, s'il dure, cesse d'être considéré comme tel. C'est pourquoi les apparitions disparaissent si vite $\rangle^{23}$. Quando, infatti, Henriette va a trovare Guillaume al fronte e recita in una pièce per sollevare l'umore dei soldati, la giovane principessa è per Thomas inizialmente come un'apparizione, ma non appena diventa troppo reale, perde tutto il suo fascino, Guillaume crede di non esserne più innamorato ed il suo interesse si rivolge nuovamente verso le eccitanti imprese militari. Non c'è spazio, dunque, per il sentimento vero nel romanzo di Cocteau, l'unica possibilità riservata all'amore è l'esistenza in una dimensione immaginaria:

Peu à peu, comme les presbytes qui ne lisent qu'à distance, Guillaume lut ses sentiments pour Henriette. Elle était loin, irréelle, factice. Elle pouvait donc entrer dans sa fiction. [...] Guillaume, torturé d'amour, ne faisait rien qui pût avertir Henriette, donner la moindre racine à son rêve ${ }^{24}$.

Questo l'atteggiamento del sognatore Guillaume che si serve di un'immagine fittizia, quella di Henriette, per alimentare la sua finzione, un gioco che per continuare ad essere non può in nessun modo radicarsi nella realtà. Gli unici personaggi divorati da una passione non fittizia soffrono, come il dottor Pesquel-Duport, o provano sentimenti malvagi, come Mme Valiche.

Il giovane Thomas vive per la prima volta totalmente la sua esperienza d'amore non accanto ad Henriette, ma nel gelido abbraccio della notte, testimone dell'ardua impresa che si conclude con la sua morte: «La nuit froide était constellée de fusées blanches et d'astres. Guillaume s'y trouvait, pour la première fois, seul. Un dernier rideau se lève. L'enfant et la féerie se confondent. Guillaume connaît enfin l'amour» ${ }^{25}$. Completamente solo sulla scena in cui si rappresenta la vita come la finzione, Guillaume recita l'ultimo atto della propria esistenza, ponendo fine alla metafora teatrale che ha costellato tutto il romanzo. Il fanciullo e l'incantesimo si confondono in un'unica immagine: questo è l'amore per Thomas, è attraverso l'estrema esaltazione di sé nella morte che Guillaume raggiunge l'apoteosi finale.

In un contesto in cui il fraintendimento e la non comprensione dilagano fra i personaggi, Henriette è innamorata di un «fantôme», la princesse de Bormes non vede che l'amore manifestato dalla figlia non è corrisposto, i soldati non comprendono la rappresentazione cui assistono, la dimensione tragica accostabile a quella del teatro è un elemento fondamentale, in quanto l'impostura qui agisce come la faute nella tragedia: una volta innescato il meccanismo dell'inganno, l'eroe viene inevitabilmente trascinato in un tragico epilogo. La menzogna fagocita, pertanto, colui che l'ha creata.

(21)J. Cocteau, Thomas l'imposteur, in Euvres romanesques complètes, cit., p. 401.

(22) Ibid.
(23) Ibid., p. 416.

(24) Ibid., p. 410.

(25) Ibid., pp. 428-429. 
Il giovane Thomas diventa l'espressione della reazione umana all'assurdità della vita ed il teatro della guerra il luogo ideale in cui liberare la più inebriante delle illusioni: «À ce vaste mensonge de sable et de feuilles, il ne manquait que Guillaume de Fontenoy» ${ }^{26}$.

Attraverso modalità e prospettive differenti, Montherlant e Cocteau conducono la loro riflessione sull'assurdità del vivere, utilizzando i temi comuni della finzione e della guerra. Se Alban de Bricoule è immerso in una persistente visione nichlilistica dell'esistenza, in cui la feinte e l'affermazione del sé costituiscono i principali punti di riferimento, Guillaume Thomas vive nell'indistinzione tra finzione e realtà e trova nella morte la massima espressione della propria identità. Per Alban la finzione è uno strumento del vivere. Per Guillaume è l'espressione della propria esistenza. Per entrambi, naturalmente collocati agli antipodi della società convenzionale, l'illusione della guerra è negativa quando è imposizione e manipolazione altrui, positiva quando è gioco, fantasia, esaltazione personale, parte inalienabile della propria verità.

PIERANGELA ADINOLFI

(26) Ibid., p. 406. Sull'opera di Jean Cocteau, segnaliamo i seguenti studi: S. LiNARES, Cocteau. La ligne d'un style, Paris, Sedes, 2000; B. Du CHAmbon,
Le Roman de Jean Cocteau, Paris, L'Harmattan, 2002; C. Arnaud, Jean Cocteau, Paris, Gallimard, 2003. 